\title{
Cell Senescence in Myxoid/Round Cell Liposarcoma
}

\section{Christina Kåbjörn Gustafsson, ${ }^{1}$ Anders Ståhlberg, ${ }^{1}$ Katarina Engtröm, ${ }^{2}$ Anna Danielsson, Ingela Turesson, ${ }^{3}$ and Pierre Åman ${ }^{1}$}

\author{
${ }^{1}$ Sahlgrenska Cancer Center, Department of Pathology, Institute of Biomedicine, University of Gothenburg, Box 425, \\ 40530 Gothenburg, Sweden \\ ${ }^{2}$ Department of Oncology, Institute of Medical Sciences, University of Gothenburg, Blå stråket 2, 41345 Gothenburg, Sweden \\ ${ }^{3}$ Department of Oncology, Institute of Medical Sciences, Uppsala University, 75185 Uppsala, Sweden
}

Correspondence should be addressed to Pierre Åman; pierre.aman@gu.se

Received 21 January 2014; Revised 22 May 2014; Accepted 23 May 2014; Published 24 June 2014

Academic Editor: Charles Catton

\begin{abstract}
Copyright ( $\odot 2014$ Christina Kåbjörn Gustafsson et al. This is an open access article distributed under the Creative Commons Attribution License, which permits unrestricted use, distribution, and reproduction in any medium, provided the original work is properly cited.
\end{abstract}

\begin{abstract}
Myxoid/round cell liposarcoma (MLS/RCLS) is the second most common liposarcoma type and characterized by the fusion oncogenes FUS-DDIT3 or EWSR1-DDIT3. Previous analysis of cell cycle regulatory proteins revealed a prominent expression of G1cyclins, cyclin dependent kinases, and their inhibitors but very few cells progressing through the G1/S boundary. Here, we extend the investigation to proteins involved in cell senescence in an immunohistochemistry based study of 17 MLS/RCLS cases. Large subpopulations of tumor cells expressed the RBL2 pocket protein and senescence associated heterochromatin $1 \gamma$ and IL8 receptor $\beta$. We conclude that MLS/RCLS tissues contain major populations of senescent tumor cells and this may explain the slow growth rate of this tumor type.
\end{abstract}

\section{Introduction}

Liposarcoma is the most common soft tissue tumor in humans and myxoid/round cell liposarcoma (MLS/RCLS) is the second most common liposarcoma type. In a majority of the cases MLS/RCLS develops in large muscles, most often in the thigh [1]. MLS/RCLS tissue is composed of round to oval shaped mesenchymal cells and a variable number of lipoblasts, set in a myxoid matrix with a fine piped capillary network. Most cases of MLS tumors are relatively slow growing but $10-15 \%$ show a hyper cellular round cell morphology (RCLS) with less myxoid component that is associated with an unfavourable prognosis [1].

The fusion oncogenes FUS-DDIT3 or EWSR1-DDIT3 are present in almost all cases of MLS/RCLS. They result from $t(12 ; 16)$ or $t(12 ; 22)$ chromosome translocation and have causative roles in development of MLS/RCLS [2-5]. FUSDDIT3 and EWSR1-DDIT3 encode abnormal transcription factors that deregulate the expression of target genes [6-8].
For example, we previously showed that FUS-DDIT3 directly induces production of IL6 and IL8 by binding to the IL 6 and IL8 promoters $[9,10]$.

A recent study reports that most MLS/RCLS cases also carry TERT promoter mutations, suggesting an increased TERT activity and extended cellular life span [11]. The RCLS variant is also associated with additional mutations, most commonly in PIK3CA [12].

Previous studies of cell cycle regulating proteins in MLS/RCLS revealed a prominent expression of growth promoting G1 cyclins and cyclin dependent kinases (CDK), coexpressed with cyclin dependent kinase inhibitors P16, P19, and P27 (also known as CDKN2A, CDKN2D, and CDKN1B, resp.) [13]. This pattern suggested that the growth promoting cyclin/CDK activity was counteracted by CDK inhibitors and this could explain the low frequency of proliferating cells identified by Ki67 and cyclin A staining (typically less than 8 and 4 percent, resp.) [13].

Recent results from investigations of senescent cells suggest that they express G1 cyclins, CDKs, and their inhibitors 
TABLE 1: Cases and immunohistochemistry data.

\begin{tabular}{|c|c|c|c|c|c|c|c|c|c|c|}
\hline Case & Age & & Site & $\mathrm{RB1}^{1}(\%)$ & $\mathrm{HP} 1 \gamma^{1}(\%)$ & $\mathrm{RBL}^{1}(\%)$ & $\mathrm{IL}^{1} \mathrm{R}^{1}(\%)$ & $\mathrm{KI}^{1} 7^{1}(\%)$ & $\begin{array}{c}\text { FUS/DDIT3 } \\
\text { rearrangement }{ }^{2}\end{array}$ & $\begin{array}{l}\text { Histological } \\
\text { classification }\end{array}$ \\
\hline 1 & 42 & im & Hip & 35 & 50 & 79 & 55 & nd & + & MLS \\
\hline 2 & 45 & $\mathrm{im}$ & Thigh & 23 & 66 & 77 & 86 & $<1$ & + & MLS \\
\hline 3 & 37 & $\mathrm{im}$ & Thigh & 73 & 50 & 69 & 69 & 4 & + & MLS \\
\hline 4 & 36 & $\mathrm{im}$ & Thigh & 60 & 56 & 55 & 86 & $<1$ & + & MLS \\
\hline 5 & 80 & im & Thigh & 62 & 60 & 93 & 91 & 2 & + & MLS \\
\hline 6 & 34 & im & Thigh & 58 & 44 & 91 & 79 & 3 & + & MLS \\
\hline 7 & 56 & im & Thigh & 72 & 47 & 76 & 64 & $<1$ & + & MLS \\
\hline 8 & 46 & $\mathrm{sc}$ & Thigh & 65 & 51 & 85 & 86 & 1 & + & MLS \\
\hline 9 & 73 & $\mathrm{im}$ & Lower leg & 75 & 49 & 83 & 83 & $<1$ & nd & MLS \\
\hline 10 & 45 & other & Peritoneal & 73 & 54 & 80 & 80 & 3 & nd & MLS \\
\hline 11 & 49 & $\mathrm{sc}$ & Thorax met & 94 & 56 & 93 & 90 & nd & + & MLS \\
\hline 12 & 33 & other & Peritoneal & 25 & 76 & 87 & 50 & $<1$ & + & MLS/RCLS \\
\hline 13 & 37 & im & Leg & 9 & 14 & 69 & 62 & nd & nd & MLS/RCLS \\
\hline 14 & 38 & $\mathrm{im}$ & Thigh & 81 & 47 & 68 & 85 & 6 & + & MLS/RCLS \\
\hline 15 & 76 & $\mathrm{sc}$ & Back & 73 & 50 & 82 & 54 & $<1$ & + & MLS/RCLS \\
\hline 16 & 46 & $\mathrm{im}$ & Hip & 85 & 45 & 0 & 74 & 8 & + & RCLS \\
\hline 17 & 19 & im & Shoulder & 77 & 51 & 78 & 79 & 7 & nd & RCLS \\
\hline
\end{tabular}

${ }^{1}$ Percent cells expressing respective protein.

${ }^{2}$ FUS-DDIT3 rearrangement status as determined by FISH. ${ }^{+}$Indicates presence of FUS-DDIT3 rearrangements. nd: not determined due to technical problems or lack of material.

im: intramuscular; sc: subcutaneous; nd: not determined; met: metastasis.

as well as a typical expression signature of cytokines and their receptors [14-17]. These new data and concepts provide a possible explanation for the expression patterns observed in MLS/RCLS and prompted a renewed investigation with focus on senescence markers. In the present study, we have employed immunohistochemistry (IHC) and analysed a cohort of 17 MLS/RCLS cases for expression of proteins associated with growth control and senescence. The results suggest that substantial proportions of MLS/RCLS tumor cells are senescent.

\section{Materials and Methods}

2.1. Tissue Samples. The clinicopathological characteristics of all tumors are presented in Table 1. Formalin-fixed tissues taken at surgery from previously untreated cases with MLS/RCLS were embedded in paraffin using routine procedures and stored at room temperature. Use of tissue samples for this study was examined and approved by the Ethical Board associated with the University of Gothenburg. All selected MLS cases were examined by two clinical pathologists specialized in soft tissue tumors and by the first author, also a clinical pathologist.

2.2. Cell Cultures. Human foreskin derived fibroblasts (passage 8 ) were cultured in RPMI1640 with 5\% fetal calf serum. Immunofluorescence studies were made 5 days after a $10 \mathrm{~Gy}$ dose of X-ray radiation. MLS cell lines 402-91, 1765-92, and 2645-94 and HT1080 clones [4, 18, 19] were cultured in RPMI1640 with GlutaMAX and 8\% fetal bovine serum, $100 \mathrm{U} / \mathrm{mL}$ penicillin, and $100 \mu \mathrm{g} / \mathrm{mL}$ streptomycin. All media and supplements were obtained from Life Technologies. All cells were maintained at $37^{\circ} \mathrm{C}$ with air containing $5 \% \mathrm{CO}_{2}$.

2.3. IL-6 Dependence Assay. MLS cell lines 402-91, 176592, and 2645-94 were seeded to 96 well microtiter plates at 2000 cells/well and allowed to settle for 8 hours. The culture medium was replaced with medium supplemented with $3 \%$ fetal bovine serum, with and without 1 unit $/ \mathrm{mL}$ of recombinant IL6 and with or without $0.5 \mu \mathrm{g} / \mathrm{mL}$ of monoclonal IL6 antibody mAB 206 (R\&D systems). Trypan blue excluding cells were counted in an inverted microscope after 48 hours of incubation. An Epstein-Barr virus immortalized lymphoblastoid cell line was included as positively responding control [20].

2.4. Immunohistochemistry and Immunofluorescence Microscopy. Series of $5 \mu \mathrm{m}$ tissue sections were cut from each biopsy, deparaffinised, rehydrated, boiled in microwave oven for 10 minutes for epitope retrievement, and stained with the antibodies listed in Table 2. Bound antibodies were visualized using the LSAB secondary antibody streptavidin biotin peroxidase system (DAKO). Stained sections were examined on a light microscope.

For immunofluorescence analysis, cultures of human fibroblasts were washed twice with PBS and fixed in $4 \%$ 
TABLE 2: Antibodies used for immunohistochemistry and immunofluorescence.

\begin{tabular}{lccc}
\hline Antigen & Antibody & Dilution & Retrievement \\
\hline IL8R $\beta$ & SC-23811 & $1: 50$ & PH6 \\
RBL2 & SC-53641 & $1: 50$ & PH9 \\
RB1 & BD \#5544136 & $1: 20$ & PH9 \\
TP53 & Calbio.OP43A & $1: 100$ & \\
CCNA & NM MS1061-S1 & $1: 15$ & \\
P21 & Millipore OP64 & $1: 25$ & \\
P15 & SC-56327 & $1: 20$ & \\
HP1 $\gamma$ & Millipore 05-690 & $1: 100$ & PH6 \\
KI67 & Dako IR-626 & $1: 1$ & PH6 \\
\hline
\end{tabular}

SC: SantaCruz Biotechnology; BD: Becton Dickinson and Company; Calbio: calbiochem; NM: neomarker; epitope retrievement was performed at indicated PH by boiling for 5 minutes.

paraformaldehyde in PBS. After two more washes in PBS, the slides were mounted in an antifade mount containing the DNA binding dye DAPI (4,6-diamidino-2-phenylindole dihydrochloride) (Olink Bioscience) and examined on a fluorescence microscope. Several antibodies were tested for each antigen and evaluated by IHC staining of tissue sections containing published positive and negative cell populations. The selected primary antibodies were also tested by western blot analysis of MLS cell lines and other reference cell lines as described elsewhere [13]. Irradiated fibroblasts were used as a control for the senescence markers used (Table 3). Detection of RB1 expression worked satisfactorily only with the antibody and protocol detailed in Table 2. Evaluation of IHC stains was made by Christina Kåbjörn, specialist pathologist, and Pierre Åman.

2.5. Flow Cytometric Analysis of Cell Cycle Phase Distribution. Paraffin-embedded, formalin-fixed tissues were dewaxed, and single-cell suspensions were prepared and labeled with propidium iodide (Life Technology, catalogue number P3566) according to the providers protocol and as previously described [21]. Flow cytometric analysis of DNA content was evaluated using the FACS calibur system (BD Biosciences). The ModFit LT software (Verity Software House) was used for analysis and peak detection to identify distributions of G1, S, and G2 cells.

2.6. FISH Analysis. Interphase FISH analysis of FUS-DDIT3 and EWSR1-DDIT3 rearrangements [22] was performed on formalin-fixed 1-4 $\mu \mathrm{m}$ paraffin tissue sections. Three breakapart probes, DDIT3, FUS, and EWSR1 (Vysis Inc.), were used according to protocols supplied by the manufacturer. Nuclei were counterstained with $10 \mu \mathrm{L} 4^{\prime}, 6^{\prime}$,-diamidino-2' phenylindole dihydrochloride (DAPI). The sections were analyzed and reanalyzed by two independent reviewers. At least 100 nuclei per section were scored. The interpretation of intact, fusion, and split signals was based on guidelines recommended by the manufacturer and from other clinical laboratories using this method.
TABLE 3: Senescence markers in irradiated fibroblasts.

\begin{tabular}{lcc}
\hline Marker & Control & Irradiated \\
\hline IL8R & Negative & $100^{++}$ \\
RBL2 & $8,12$ (mitotic) $)^{++}$ & $94,96^{+}$ \\
RB1 & $100^{++}$ & $30,33^{+}$ \\
P53 & Negative & $100^{++}$ \\
CCNA & $17,20^{++}$ & $N^{+}$ \\
P21 & Negative \\
P15 & Negative & $100^{+++}$ \\
HP1 $\gamma$ & $<1^{++}$ & $100^{+}$ \\
Percentage positive cells for respective marker are shown. ${ }^{++++,+++}$indicate \\
weak, medium, and strong expression, respectively. Mitotic: expression seen \\
only in mitotic cells. Results from two experiments are shown. Two values \\
are shown when there was variation between the experiments.
\end{tabular}

\section{Results}

Irradiated fibroblasts were used as a control for the senescence markers and antibodies used. Our data showed a very strong expression of all investigated senescence associated markers in irradiated cells compared to control cultured cells (Table 3).

Our Ki67 IHC staining results (Table 1) and previous data [13] showed that most MLS cases contain very few Ki 67 positive cells, typically less than $4 \%$, but some RCLS tissues contained smaller tissue regions with up to $8 \%$ positive cells. A previous investigation also showed only few cells expressing the S-G2 phase specific cyclin A, suggesting that a majority of the cells are arrested in the G1 phase [13]. Here, our flow cytometry analysis of cell nuclei from two human MLS/RCLS tissues showed 94\% and 96\% G1-phase cells, respectively (Figure 1), supporting our conclusion that most MLS/RCLS tumor cells were arrested in the G1 phase of the cell cycle.

IHC analysis of pocket proteins showed that 9-94\% of the cells expressed the proliferation associated RB1 and 0-93\% expressed the rest of phase protein RBL2. It is thus obvious that there are overlaps in expression of the RB1 and RBL2 proteins.

Our IHC analysis showed only weak signals for the RB1 protein in MLS tissues compared to control tissue samples from other tumor types. This prompted a further analysis of RB1 protein expression in MLS cells. Western blot analysis showed a strong expression of a normal sized RB1 protein in all three investigated MLS derived cell lines (Figure 2). Forced expression of FUS-DDIT3 gave no effects on RB1 expression in the human HT1080 fibrosarcoma cell line (Figure 2).

Between 14 and $76 \%$ of the tumor cells stained positive for heterochromatin protein (HPl $\gamma$ ) (Table 1 and Figure 1). Together with the pocket protein results, these data suggest that a large proportion of the MLS/RCLS cells are arrested in the G1 phase and that the tumors contain a substantial fraction of senescent cells.

Cell senescence is associated with a distinct cytokine and cytokine receptor expression signature and FUS-DDIT3 is known to induce IL6 and IL8 expression in the tumor cells. IHC analysis of MLS/RCLS tissues showed that the 


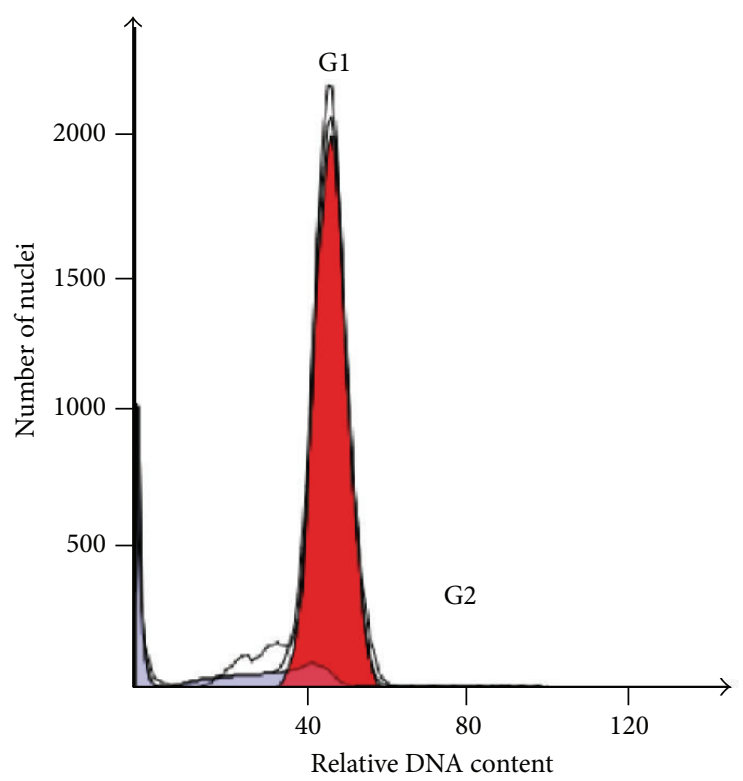

(a)
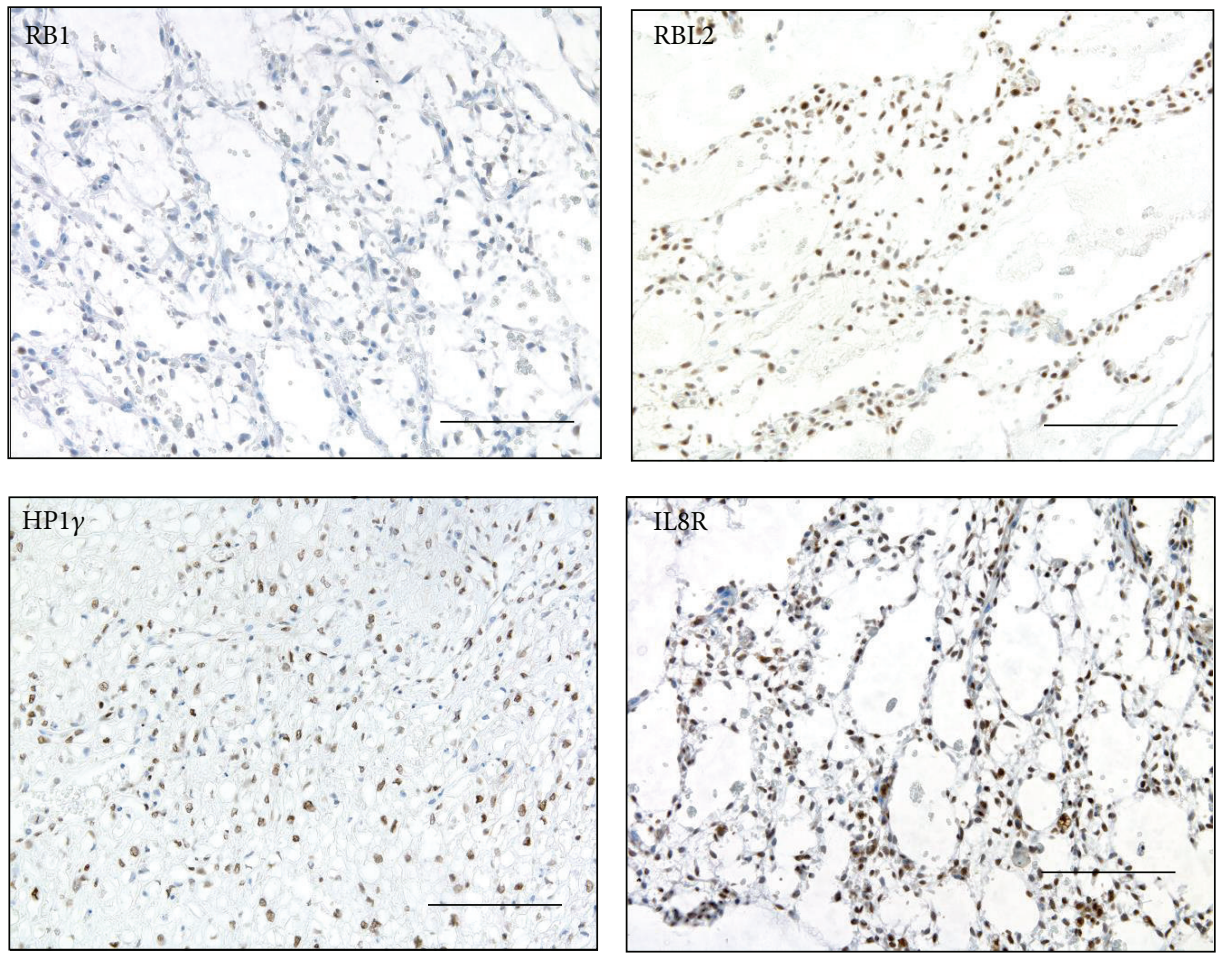

(b)

FIGURE 1: (a) Flow cytometry histogram of cell nuclei extracted from paraffin embedded MLS/RCLS tumor tissue (Cases 8) showing more than $95 \%$ of the cells in the G1 phase of the cell cycle (red). (b) Immunohistochemistry analysis of RB1, RBL2, HP1 $\gamma$, and IL8R in MLS/RCLS tumor tissues. Brown staining shows reactivity with the specific antibodies. Bars are $100 \mu \mathrm{m}$.

senescence associated IL8 receptor beta (also known as CXCR2) was expressed in 50-91\% of the tumor cells (Table 1 and Figure 1). We tested MLS/RCLS tumor cell lines for in vitro growth and survival dependence on IL6, but no effects of treatment with IL6 or IL6 blocking antibodies were detected (data not shown).

\section{Discussion}

Our previous analysis of cell cycle regulator expression in MLS/RCLS suggested that a majority of the tumor cells were arrested in the G1 phase of the cell cycle [13]. Only a few percent of the cells escape this arrest as detected 
by low numbers of Ki67 and cyclin A positive cells. This conclusion is supported here by the present Ki67 IHC analysis of 17 MLS/RCLS cases and by flow cytometry analysis of two cases.

The Ki67 and cytometry analysis was complemented by an IHC based investigation of the pocket proteins RB1 and RBL2 (also known as P105 and P130). RB1 and RBL2 are important control hubs for cell cycle regulation and proliferation driving transcription factors. $\mathrm{RB} 1$ is expressed in proliferating cells and is also necessary for induction of senescence, while RBL2 is expressed in resting and senescent cells. Consequently, the levels of RB1 decrease and the levels of RBL2 increase, as cells enter a nonproliferative or senescent cell state [23-27]. Our IHC results for RB1 and RBL2 support the previous conclusion that large subpopulations of tumor cells are in a resting state.

IHC staining of RB1 in MLS/RCLS tissues gave generally weak signals calling for further analysis. Six different RB1 specific antibodies and various staining/antigen retrieving conditions were tested (data not shown). Compared to the staining intensities for RBL2 and HP1 $\gamma$, the signal was always found weaker in MLS tumor tissues and RB1 staining was also weaker in MLS tissues compared to reference tumor tissues of other entities. Analysis of MLS/RCLS derived cell lines showed RB1 protein expression in level with other tumor cell lines and much stronger than the expression in cultured normal fibroblasts (Figure 2). Comparison of HT1080 fibrosarcoma cells with and without the FUS-DDIT3 showed that the fusion protein has no direct effect on RB1 expression (Figure 2). Taken together, our data suggest that MLS/RCLS cells are capable of normal expression of RB1. The fading RB1 expression in MLS/RCLS may thus result from a normal downregulation in connection with growth cessation of many tumor cells.

Some senescent cell types are characterized by nuclear heterochromatin foci that can be visualized by DNA-stains and the increased expression of heterochromatin protein 1 gamma $(\mathrm{HPl} \gamma)[28,29]$. The IHC analysis of HP1 $\gamma$ in MLS/RCLS tissues showed a heterogeneous pattern with large numbers of strongly stained cells. This suggests that large subpopulations of the tumor cells are senescent with expanded heterochromatin formation and thus they may be permanently excluded from further proliferation.

FUS-DDIT3 binds the promoter regions of the IL 8 and IL6 encoding genes leading to expression of these genes [9, 10]. IL6 is reported as an autocrine growth or survival factor in several tumor types [30-33]. Our results suggested, however, that IL6 is not a growth/survival factor for MLS/RCLS cells. More recently IL6 and IL8 were reported to be parts of a cytokine expression profile (IL6 and IL8) that is typical for senescent cells [34-36]. Instead of acting as growth factors, IL6 together with IL8 may thus be part of a senescence mechanism in MLS/RCLS. Our data showing IL8 receptor expression in many of the tumor cells is in line with this interpretation. The IL8 receptor beta expression also indicates a possible senescence associated IL8 autocrine activity as the tumor cells also are producing IL8 [7]. A schematic presentation of the investigated senescence associated factors in MLS/RCLS is shown in Figure 3.

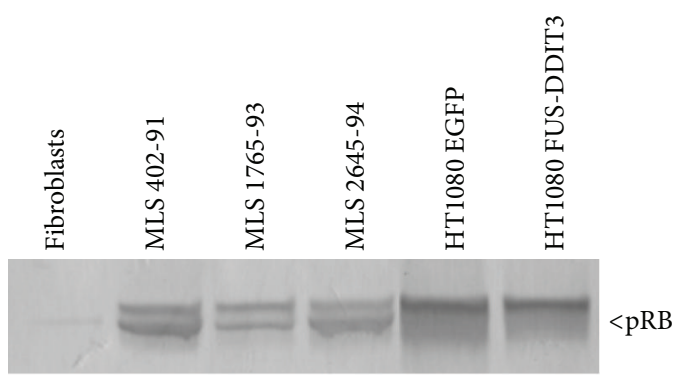

FIgURE 2: Western blot analysis of RB1 expression in cultured normal human fibroblasts, MLS cell lines, and FUS-DDIT3 or EGFP transfected HT1080 cells.

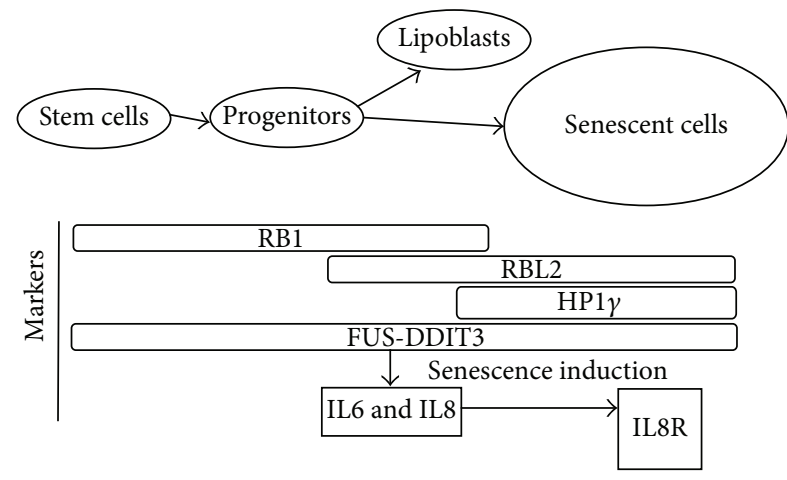

FIGURE 3: Schematic presentation of tumor populations and factors involved in senescence. A small population of proliferating cells arises from a hypothetical tumor stem cell population. Most of these cells enter senescence and a few percent differentiates into lipoblasts. RB1 and RBL2 proteins are central factors involved in growth regulation and entry/maintenance of cell senescence. RB1 is expressed in proliferating cells and is also necessary for differentiation and senescence. RBL2 is expressed in resting and senescent cells. Increased HP1 $\gamma$ expression is typical for senescent cells. IL6 and IL8 are produced by the tumor cells and IL8 may bind to the IL $8 \beta$ receptor expressed by senescent tumor cells.

Our hypothesis that major subpopulations of MLS/RCLS cells are senescent may seem contradictory to the recent report that MLS/RCLS tumors carry TERT promoter mutations. Such mutations may indicate an increased TERT activity and immortalization of the tumor cells [11]. The large numbers of senescent cells in MLS/RCLS tumors may, however, be caused by oncogene induced stress responses resulting in senescence. This hypothesis is supported by our previous in vitro experiments with FUS-DDIT3 transfection into various cell types. Forced FUS-DDIT3 expression caused cell death and senescence in most cell types and only very few cells in permissive cell lines maintained proliferative capacity [18]. Surviving FUS-DDIT3 transfected cells also had a slower in vivo and in vitro growth rate compared to wild-type cells [18]. Oncogenic stress induced senescence has been reported for many oncogenes and is thought to serve as a major barrier against tumor development in vivo [14-17]. This mechanism may also explain the observed low growth rate and abundance of senescent cells in MLS/RCLS tumors. 
Ten to fifteen percent of MLS/RCLS cases show round cell morphology and this is associated with increased growth rate and unfavorable prognosis. Inspection of our limited cohort of cases failed to detect any correlation between the RCLS morphology and expression data of the analyzed markers. A possible association between RCLS morphology and number of senescent cells has to be tested in a larger tumor material.

Irradiated fibroblasts showed a very strong expression of all investigated senescence associated markers. In a clinical study, we have previously reported that irradiation of MLS/RCLS tumors results in transformation to a lipoma like morphology with highly differentiated adipocyte like cells [37]. Further investigations of irradiated MLS/RCLS tumors will show if this treatment causes maturation of senescent cells into adipocyte like cells. Such effects have been reported in other tumor types [38].

We have investigated 17 MLS/RCLS tumors for expression of senescence associated proteins. The results suggest that large subpopulations of tumor cells are in a senescent cell state characterized by expression of $\mathrm{HP} 1 \gamma, \mathrm{RBL} 2$, and senescence associated cytokines and a cytokine receptor. The presence of large numbers of senescent cells may explain the observed slow growth rate of this sarcoma type.

\section{Conflict of Interests}

None of the authors have any economical or other conflicting interests with regard to the publication of this paper.

\section{Acknowledgments}

This work was supported by grants from the Swedish Cancer Society, Swedish Research Council, LUA/ALF grants, Västra Götaland, Assar Gabrielssons Research Foundation, Johan Jansson Foundation for Cancer Research, Swedish Society for Medical Research, BioCARE National Strategic Research Program at University of Gothenburg, and Wilhelm and Martina Lundgren Foundation for Scientific Research.

\section{References}

[1] C. D. Fletcher, K. K. Unni, and F. Mertens, Tumors of Soft Tissue and Bone. WHO Classification Pathology and Genetics, IARCPress, Lyon, France, 2000.

[2] A. Crozat, P. Aman, N. Mandahl, and D. Ron, "Fusion of CHOP to a novel RNA-binding protein in human myxoid liposarcoma," Nature, vol. 363, no. 6430, pp. 640-644, 1993.

[3] T. H. Rabbitts, A. Forster, R. Larson, and P. Nathan, "Fusion of the dominant negative transcription regulator $\mathrm{CHOP}$ with a novel gene FUS by translocation $\mathrm{t}(12 ; 16)$ in malignant liposarcoma," Nature Genetics, vol. 4, no. 2, pp. 175-180, 1993.

[4] P. Åman, D. Ron, N. Mandahl et al., "Rearrangement of the transcription factor gene CHOP in myxoid liposarcomas with t(12;16)(q13;p11)," Genes Chromosomes and Cancer, vol. 5, no. 4, pp. 278-285, 1992.

[5] I. Panagopoulos, M. Höglund, F. Mertens, N. Mandahl, F. Mitelman, and P. Åman, "Fusion of the EWS and CHOP genes in myxoid liposarcoma," Oncogene, vol. 12, no. 3, pp. 489-494, 1996.

[6] N. Riggi, L. Cironi, P. Provero et al., "Expression of the FUSCHOP fusion protein in primary mesenchymal progenitor cells gives rise to a model of myxoid liposarcoma," Cancer Research, vol. 66, no. 14, pp. 7016-7023, 2006.

[7] H. Zinszner, R. Albalat, and D. Ron, "A novel effector domain from the RNA-binding protein TLS or EWS is required for oncogenic transformation by CHOP," Genes and Development, vol. 8, no. 21, pp. 2513-2526, 1994.

[8] H. Zinszner, D. Immanuel, Y. Yin, F.-X. Liang, and D. Ron, "A topogenic role for the oncogenic N-terminus of TLS: nucleolar localization when transcription is inhibited," Oncogene, vol. 14, no. 4, pp. 451-461, 1997.

[9] M. Göransson, M. K. Andersson, C. Forni et al., "The myxoid liposarcoma FUS-DDIT3 fusion oncoprotein deregulates NF$\kappa \mathrm{B}$ target genes by interaction with NFKBIZ," Oncogene, vol. 28, no. 2, pp. 270-278, 2009.

[10] M. Göransson, E. Elias, A. Ståhlberg, A. Olofsson, C. Andersson, and P. Åman, "Myxoid liposarcoma FUS-DDIT3 fusion oncogene induces C/EBP $\beta$-mediated interleukin 6 expression," International Journal of Cancer, vol. 115, no. 4, pp. 556-560, 2005.

[11] P. J. Killela, Z. J. Reitmana, Y. Jiao et al., "TERT promoter mutations occur frequently in gliomas and a subset of tumors derived from cells with low rates of self-renewal," Proceedings of the National Academy of Sciences of the United States of America, vol. 110, no. 15, pp. 6021-6026, 2013.

[12] J. Barretina, B. S. Taylor, S. Banerji et al., "Subtype-specific genomic alterations define new targets for soft-tissue sarcoma therapy," Nature Genetics, vol. 42, no. 8, pp. 715-721, 2010.

[13] A. Olofsson, H. Willén, M. Göransson et al., "Abnormal expression of cell cycle regulators in FUS-CHOP carrying liposarcomas," International Journal of Oncology, vol. 25, no. 5, pp. 1349-1355, 2004.

[14] M. Braig, S. Lee, C. Loddenkemper et al., "Oncogene-induced senescence as an initial barrier in lymphoma development," Nature, vol. 436, no. 7051, pp. 660-665, 2005.

[15] J. Bartkova, N. Rezaei, M. Liontos et al., "Oncogene-induced senescence is part of the tumorigenesis barrier imposed by DNA damage checkpoints," Nature, vol. 444, no. 7119, pp. 633-637, 2006.

[16] M. Braig and C. A. Schmitt, "Oncogene-induced senescence: putting the brakes on tumor development," Cancer Research, vol. 66, no. 6, pp. 2881-2884, 2006.

[17] P. Yaswen and J. Campisi, "Oncogene-induced senescence pathways weave an intricate tapestry," Cell, vol. 128, no. 2, pp. 233-234, 2007.

[18] K. Engström, H. Willén, C. Kåbjörn-Gustafsson et al., “The myxoid/round cell liposarcoma fusion oncogene FUS-DDIT3 and the normal DDIT3 induce a liposarcoma phenotype in transfected human fibrosarcoma cells," American Journal of Pathology, vol. 168, no. 5, pp. 1642-1653, 2006.

[19] S. Thelin-Jarnum, C. Lassen, I. Panagopoulos, N. Mandahl, and P. Aman, "Identification of genes differentially expressed in TLS-CHOP carrying myxoid liposarcomas," International Journal of Cancer, vol. 83, no. 1, pp. 30-33, 1999.

[20] T. Yokoi, T. Miyawaki, A. Yachie, K. Kato, Y. Kasahara, and N. Taniguchi, "Epstein-Barr virus-immortalized B cells produce IL-6 as an autocrine growth factor," Immunology, vol. 70, no. 1, pp. 100-105, 1990. 
[21] I. Köpf, C. Hanson, U. Delle, I. Verbiené, and A. Weimarck, "A rapid and simplified technique for analysis of archival formalin-fixed, paraffin-embedded tissue by fluorescence in situ hybridization (FISH)," Anticancer Research, vol. 16, no. 5, pp. 2533-2536, 1996.

[22] H. Yoshida, K. Nagao, H. Ito, K. Yamamoto, and S. Ushigome, "Chromosomal translocations in human soft tissue sarcomas by interphase fluorescence in situ hybridization," Pathology International, vol. 47, no. 4, pp. 222-229, 1997.

[23] H. Helmbold, W. Deppert, and W. Bohn, "Regulation of cellular senescence by Rb2/p130," Oncogene, vol. 25, no. 38, pp. 5257$5262,2006$.

[24] F. P. Fiorentino, C. E. Symonds, M. MacAluso, and A. Giordano, "Senescence and p130/Rbl2: a new beginning to the end," Cell Research, vol. 19, no. 9, pp. 1044-1051, 2009.

[25] S. Haferkamp, S. L. Tran, T. M. Becker, L. L. Scurr, R. F. Kefford, and H. Rizos, "The relative contributions of the p53 and pRb pathways in oncogene-induced melanocyte senescence," Aging, vol. 1, no. 6, pp. 542-556, 2009.

[26] H. Helmbold, N. Kömm, W. Deppert, and W. Bohn, "Rb2/p130 is the dominating pocket protein in the p53-p21 DNA damage response pathway leading to senescence," Oncogene, vol. 28, no. 39, pp. 3456-3467, 2009.

[27] H. Helmbold, U. Galderisi, and W. Bohn, "The switch from $\mathrm{pRb} / \mathrm{p} 105$ to Rb2/p130 in DNA damage and cellular senescence," Journal of Cellular Physiology, vol. 227, no. 2, pp. 508-513, 2012.

[28] P. D. Adams, "Remodeling chromatin for senescence," Aging Cell, vol. 6, no. 4, pp. 425-427, 2007.

[29] R. Zhang, W. Chen, and P. D. Adams, "Molecular dissection of formation of senescence-associated heterochromatin foci," Molecular and Cellular Biology, vol. 27, no. 6, pp. 2343-2358, 2007.

[30] D. Giri and M. Ittmann, "Interleukin-8 is a paracrine inducer of fibroblast growth factor 2, a stromal and epithelial growth factor in benign prostatic hyperplasia," American Journal of Pathology, vol. 159, no. 1, pp. 139-147, 2001.

[31] D. Giri, M. Ozen, and M. Ittmann, "Interleukin-6 is an autocrine growth factor in human prostate cancer," American Journal of Pathology, vol. 159, no. 6, pp. 2159-2165, 2001.

[32] M. M. Kawano, H. Ishikawa, N. Tsuyama et al., "Growth mechanism of human myeloma cells by interleukin-6," International Journal of Hematology, vol. 76, supplement 1, pp. 329-333, 2002.

[33] H. Nakanishi, K. Yoshioka, S. Joyama et al., "Interleukin6/soluble interleukin-6 receptor signaling attenuates proliferation and invasion, and induces morphological changes of a newly established pleomorphic malignant fibrous histiocytoma cell line," American Journal of Pathology, vol. 165, no. 2, pp. 471480, 2004.

[34] J. C. Acosta, A. O'Loghlen, A. Banito et al., "Chemokine signaling via the CXCR2 receptor reinforces senescence," Cell, vol. 133, no. 6, pp. 1006-1018, 2008.

[35] J. C. Acosta, A. O'Loghlen, A. Banito, S. Raguz, and J. Gil, "Control of senescence by CXCR2 and its ligands," Cell Cycle, vol. 7, no. 19, pp. 2956-2959, 2008.

[36] T. Kuilman and D. S. Peeper, "Senescence-messaging secretome: SMS-ing cellular stress," Nature Reviews Cancer, vol. 9, no. 2, pp. 81-94, 2009.

[37] K. Engström, P. Bergh, C.-G. Cederlund et al., "Irradiation of myxoid/round cell liposarcoma induces volume reduction and lipoma-like morphology," Acta Oncologica, vol. 46, no. 6, pp. 838-845, 2007.
[38] K.-H. von Wangenheim, H.-P. Peterson, and K. Schwenke, "A major component of radiation action: interference with intracellular control of differentiation," International Journal of Radiation Biology, vol. 68, no. 4, pp. 369-388, 1995. 


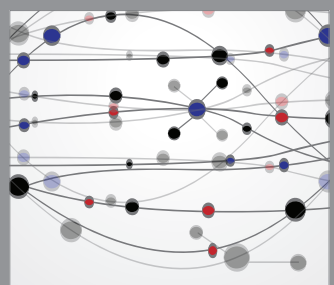

The Scientific World Journal
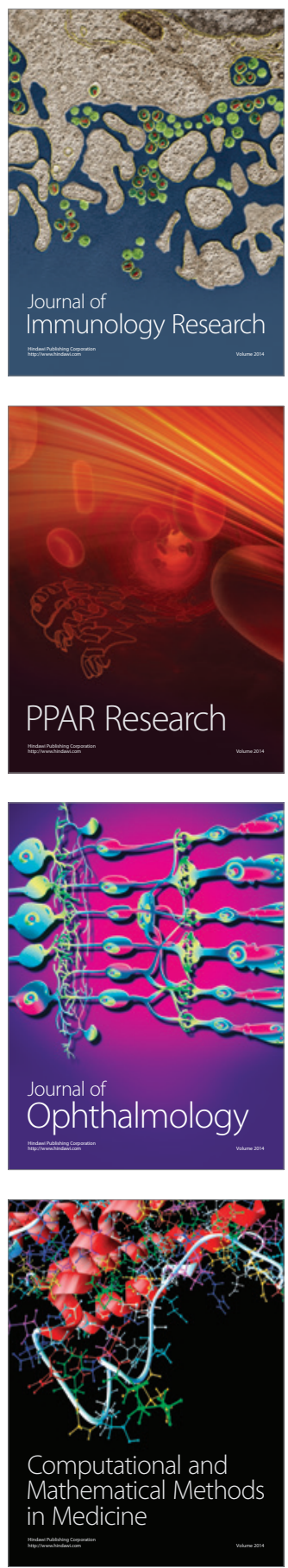

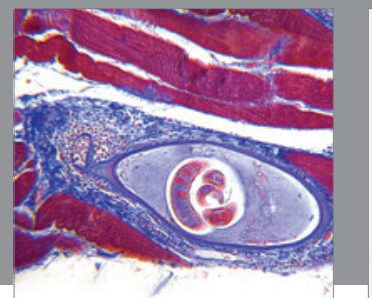

Gastroenterology

Research and Practice
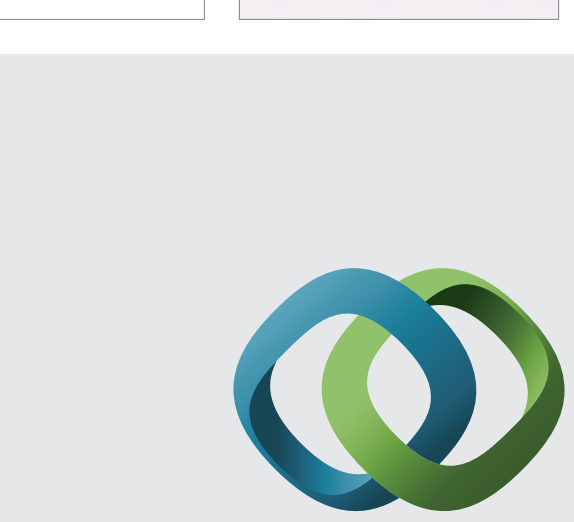

\section{Hindawi}

Submit your manuscripts at

http://www.hindawi.com
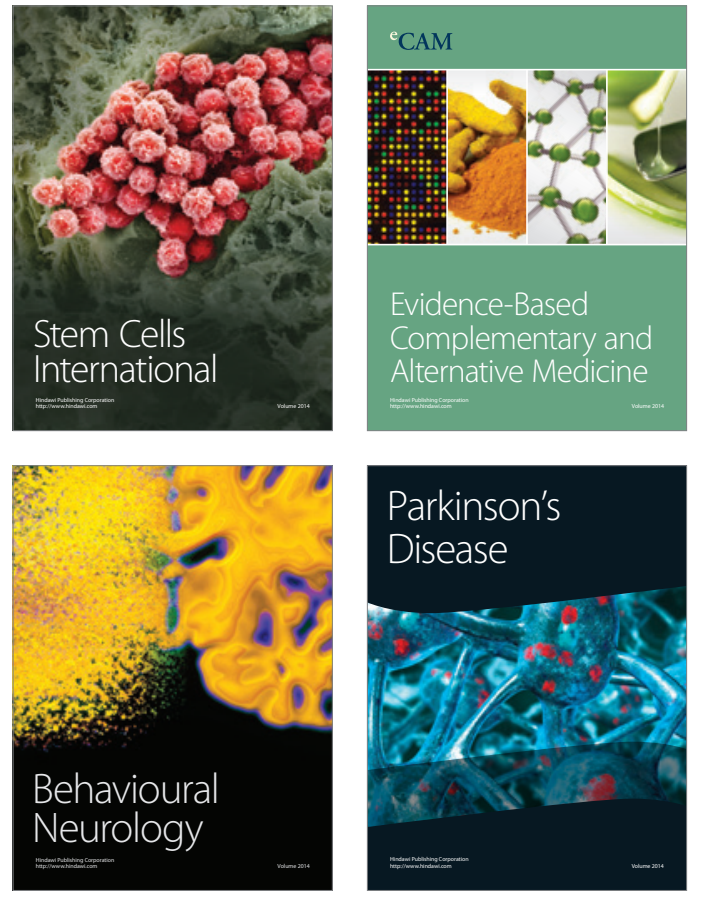
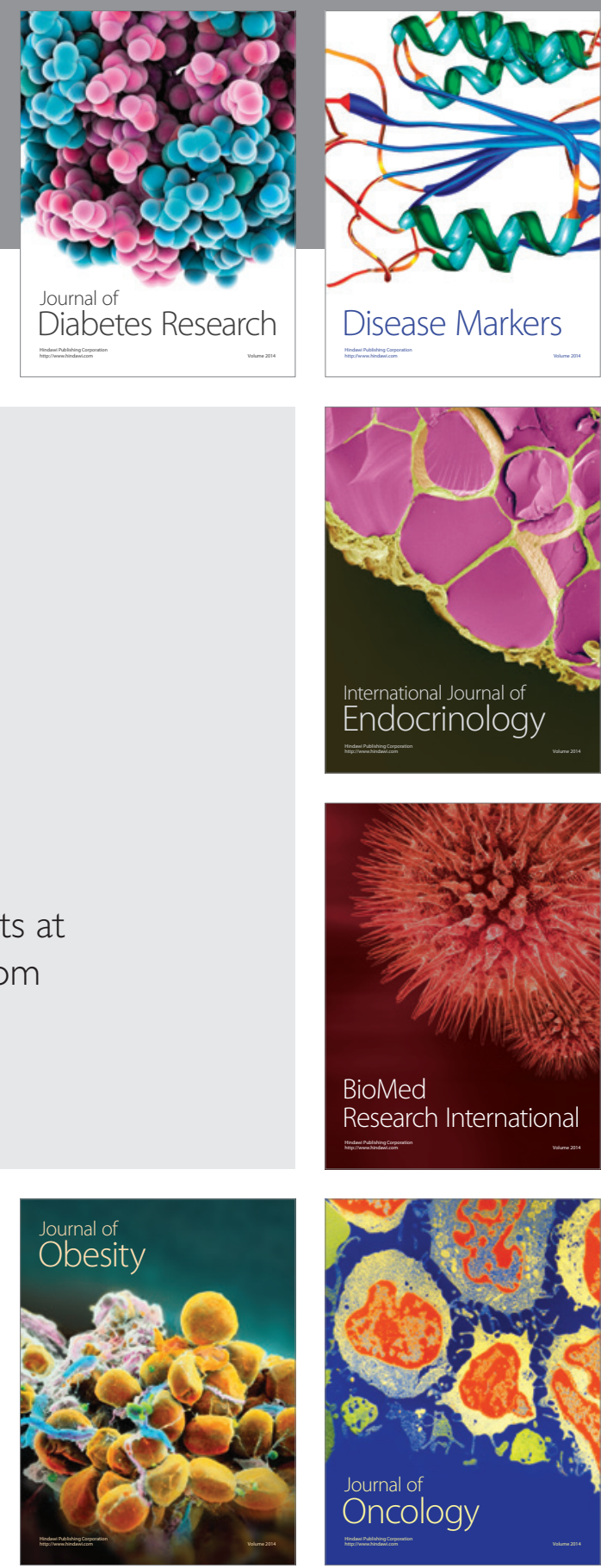

Disease Markers
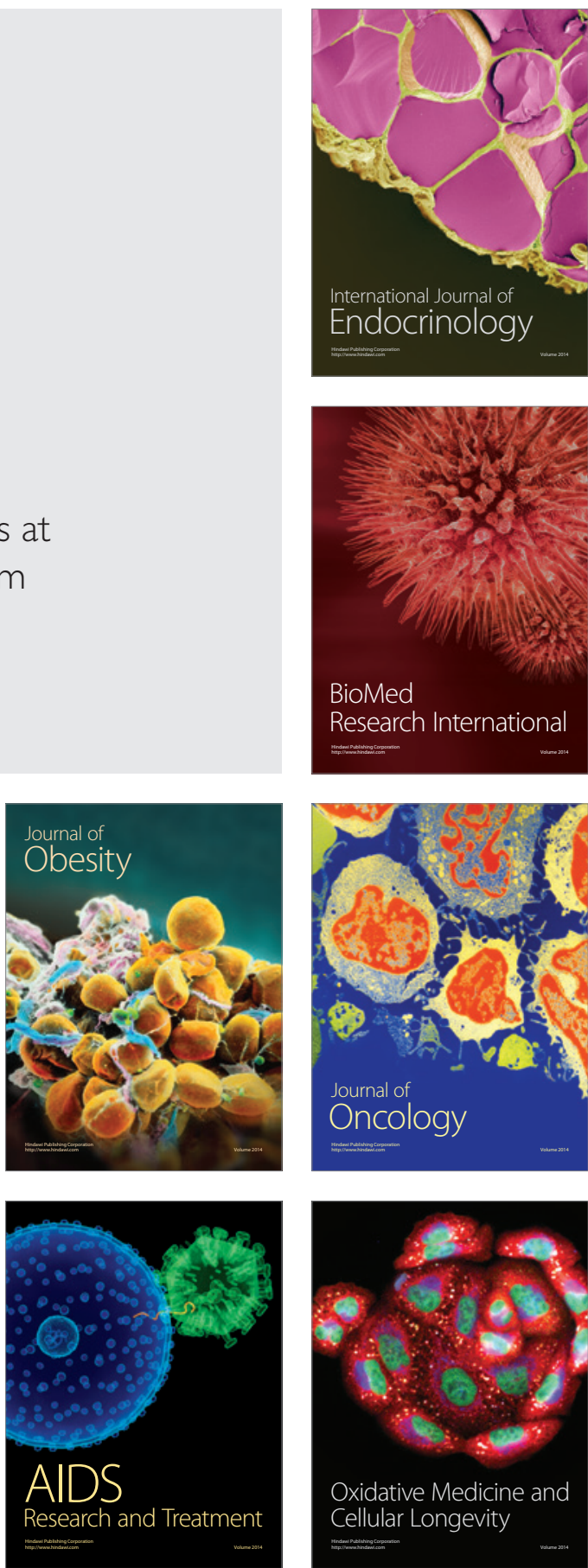